\title{
Situación de la Certificación en Organizaciones de Gestión de Instalaciones Deportivas en España
}

\author{
Situation of the Certification in Sports Facility \\ Management Organizations in Spain \\ Moisés Grimaldi-Puyana ${ }^{1}$ y Antonio Jesús Sánchez-Oliver ${ }^{2}$ \\ 1 Departamento de Educación Física y Deporte, Universidad de Sevilla (España). \\ 2 Área de Motricidad Humana y Rendimiento Deportivo de la Universidad de Sevilla, Sevilla (España). \\ Facultad de Ciencias del Deporte de la Universidad Pablo de Olavide, Sevilla (España).
}

\begin{abstract}
Resumen: El objetivo de este estudio fue analizar y describir la situación actual de la calidad en las organizaciones deportivas de gestión de instalaciones deportivas espańolas. De un total de 5.065 registradas bajo el código nacional de actividades económicas 9311, se procedió al estudio pormenorizado de cada una de las organizaciones a través de la observación sistemática, para finalmente seleccionar a 35 organizaciones con certificación de calidad ISO9001, ISO14001, OHSAS 18001y EFQM. Los resultados muestran una escasa implantación de calidad en las organizaciones de gestión de instalaciones deportivas, siendo las certificaciones con mayor implantación, el sistema de gestión de calidad (ISO 9001) y el sistema de gestión medioambiental (ISO 14001).

Palabras clave: Calidad, Certificación, Gestión Deportiva, Instalaciones deportivas.
\end{abstract}

Abstract: The aim of this study was to analyze and describe the current situation of quality in sports management organizations of Spanish sports facilities. Out of a total of 5,065 registered under the national code of economic activities 9311, a detailed study of each of the organizations was carried out through systematic observation, to finally select 35 organizations with quality certification ISO9001, ISO14001, OHSAS 18001and EFQM. The results show a low quality implantation in the management organizations of sports facilities, being the certifications with greater implementation the quality management system (ISO 9001) and environmental management system (ISO 14001).

Keywords: Quality, Certification, Sports Management, Facilities.

\section{Introducción}

La preocupación por la calidad en el tejido empresarial español es innegable, como apuntan los datos referentes al volumen de certificaciones (Santos-Vijandea y Álvarez-González, 2007). A finales del siglo pasado surgió el boom de la calidad en las empresas españolas. En sus inicios, el movimiento comenzó en Europa en empresas del sector industrial, y posteriormente se popularizó en el sector servicios, servicios financieros, servicios sociales, educación, servicios sanitarios, etcétera. El objetivo principal de la implantación de sistemas de calidad fue la mejora de la gestión interna y su imagen (Casadeus y Heras-Saizarbitoria, 2005). Para Escanciano, Miranda, Esteban, Fernández-Sánchez y Vázquez-Ordás (2003) la mejora de calidad es una de las herramientas que tienen las organizaciones a su disposición para mejorar su posicionamiento en el mercado.

De ahí, que se observan trabajos que muestran la implantación medioambiental como impacto positivo en el rendimiento financiero empresarial (Arana-Landín, Heras-Sai-

Dirección para correspondencia [Correspondence address]: Antonio Jesús Sánchez-Oliver. Área de Motricidad Humana y Rendimiento Deportivo de la Universidad de Sevilla. Sevilla. Espańa.Facultad de Ciencias del Deporte de la Universidad Pablo de Olavide. Sevilla (España).

E-mail: asanchez38@us.es zarbitoria, Ochoa-Laburu, y Andonegi-Martínez, 2004), O autores como Cruz-Gómez, Lucena-Cobos, Méndez-Rodríguez y Cáceres-clavero (2004), afirman que estar certificado en el sistema agroalimentario garantiza la seguridad alimentaria, calidad nutricional y sobre todo diferencian al producto sobre las características organolépticas y a la satisfacción del acto de alimentarse ligada a factores socioculturales.

En el sector deportivo, según Dorado-Suárez (2005) la gestión en organizaciones deportivas a través de la calidad se trata de un reto de futuro, la cual pretenda destacar y ser referente en el sector. Entendida la calidad, Gávez-Ruíz (2011) afirma que se trata de una "variable más dentro de la filosofía organizacional, y su consecución no sólo se convierte en elemento diferenciador, también supone conseguir que los servicios sean competitivos, tratando de lograr asi la satisfacción de loslas usuarios/as y, consecuentemente, altos niveles de fidelización" (p, 21).

De ahí que encontramos afirmaciones, donde se insta tanto a organizaciones privadas como públicas a gestionar a través de modelos de gestión basado en la calidad (Dorado y Gallardo, 2005). Según Molina-García (2016) "Es imprescindible en toda organización deportiva que desee regirse por estándares de excelencia, elabore una planificación estratégica donde incluya, no sólo los parámetros de la organización como el 
estudio de necesidades, sino también otros como, los de calidad, sostenibilidad y liderazgo" (p.27).

Básicamente se observa usuarios con mayor formación, y cada vez más exigentes en el sector deportivo, esto hace que las organizaciones se transformen con el fin de ser más competitivos (Espejel, Fandos, y Flavián, 2007). O lo que es lo mismo, las organizaciones deportivas están en un proceso de cambio continuo en los últimos años, donde el concepto calidad se considera una prioridad para mantener la competitividad (Llorens y Fuentes, 2000).

De igual manera Sánchez-Bujan (2004) en su manuscrito "Hacia una gestión de calidad en centros deportivos", menciona la calidad como herramienta necesaria para el gestor deportivo, la cual ayuda a conocer apropiadamente a nuestro cliente, con el fin de fidelizarlo y asegurar el éxito en la gestión. En el mismo sentido Quintanilla (2002) señala que para cumplir con una gestión de calidad es importantísimo saber los gustos y prioridades de los usuarios, o lo que es lo mismo conocer la demanda e identificar las opiniones y necesidades socio deportivas (Morales-Cevidanes, 2009).

Desde la década de los ochenta, muchos autores trabajan en la evaluación de la calidad de los servicios utilizando diversas herramientas de medida (Parasuraman, Zeithaml, y Berry, 1988). Cabe destacar instrumentos de evaluación de sistemas de calidad en servicios deportivos como el SERVQUAL (Parasuraman, Zeithaml y Berry, 1985) y SERVPERF (Cronin y Taylor, 1992), siendo los instrumentos más utilizadas en las tres últimas décadas (Barros y Gonçalves, 2009; Theodorakis, Howat, Ko y Avourdiadou, 2014; Gálvez-Ruiz, Grimaldi-Puyana, Fernández-Gavira, y Sánchez-Oliver, en prensa)

Además, es fácilmente apreciable como en todos los sectores la calidad es un término ampliamente estudiado. Referido al sector deportivo, se observa en la literatura científica múltiples documentos que hacen alusión calidad de servicio, y esta repercute en la satisfacción del servicio deportivo recibido (Afthinos, Theodorakis, y Nassis, 2005; Blázquez-Manzano y Feu-Molina, 2011; Blázquez-Manzano y Feu-Molina, 2010; Calabuig, Quintanilla, y Mundina, 2008; Calabuig,; Hernández Mendo, 2001; Mañas, Jiménez, Mayor, Martínez, y Moliner, 2008; Morales Sánchez, 2009; Morales Sánchez, Hernández Mendo, y Blanco Villaseñor, 2005; Nuviala, Ta- mayo, Iranzo, y Falcón, 2008; Nuviala, Tamayo, Nuviala, González, y Fernández, 2010; Serrano, Rial, García, y Hernández Mendo, 2010; Vila, Sánchez, y Manassero, 2009).

Mientras que, en España, como afirma Gálvez-Ruiz (2011) las investigaciones en esta línea se han centrado en determinar las dimensiones relevantes del constructo de la calidad en los servicios deportivos, a través de la evaluación de determinados programas de actividad física muy definidos o aspectos muy concretos del servicio, sin que exista actualmente unanimidad al respecto.

Sin embargo según lo indicado por Segarra-Vicéns (2017), es escasa la cultura de implantación los sistemas de gestión de calidad en organizaciones deportivas, este autor describe tres niveles de implantación, la primera de escasa implantación: cartas de servicios UNE93200, Gestión de calidad ISO9001 y sello de excelencia FFQM; la segunda de muy escasa implantación: Gestión medioambiental ISO 14001 y Verificación medioambiental EMAS; y la última de implantación casi inexistentes: Certificación de riesgos laborales OHSAS 18001 y Accesibilidad Global DALCO 170001. Teniendo en cuenta todo lo expuesto anteriormente, puede decirse que la principal finalidad de este estudio fue analizar y describir la situación actual de los certificados de calidad en organizaciones deportivas.

\section{Metodología}

Se trata de un estudio descriptivo de corte transversal, donde los datos se obtuvieron de la base de datos del Sistema de Análisis de Balances Ibéricos (SABI), estos datos son procedentes del registro mercantil, de fuentes públicas (BOE, BOP, BORME...). Se realizó un primer filtrado (Fase I), seleccionando a todas las organizaciones que pertenecían al Código Nacional de Actividad Económica 931. De estas de excluyeron las 9312-Gestión de los clubes deportivos/9313-Actividades de los gimnasios/9319 Otras actividades deportivas). Finalmente se seleccionaron las del código CNAE 9311-Gestión de instalaciones deportivas ( $N=5.065$ organizaciones), las cuales solamente el $1 \%$,se observó que contaba con algún tipo de sello de calidad (Figura 1). 


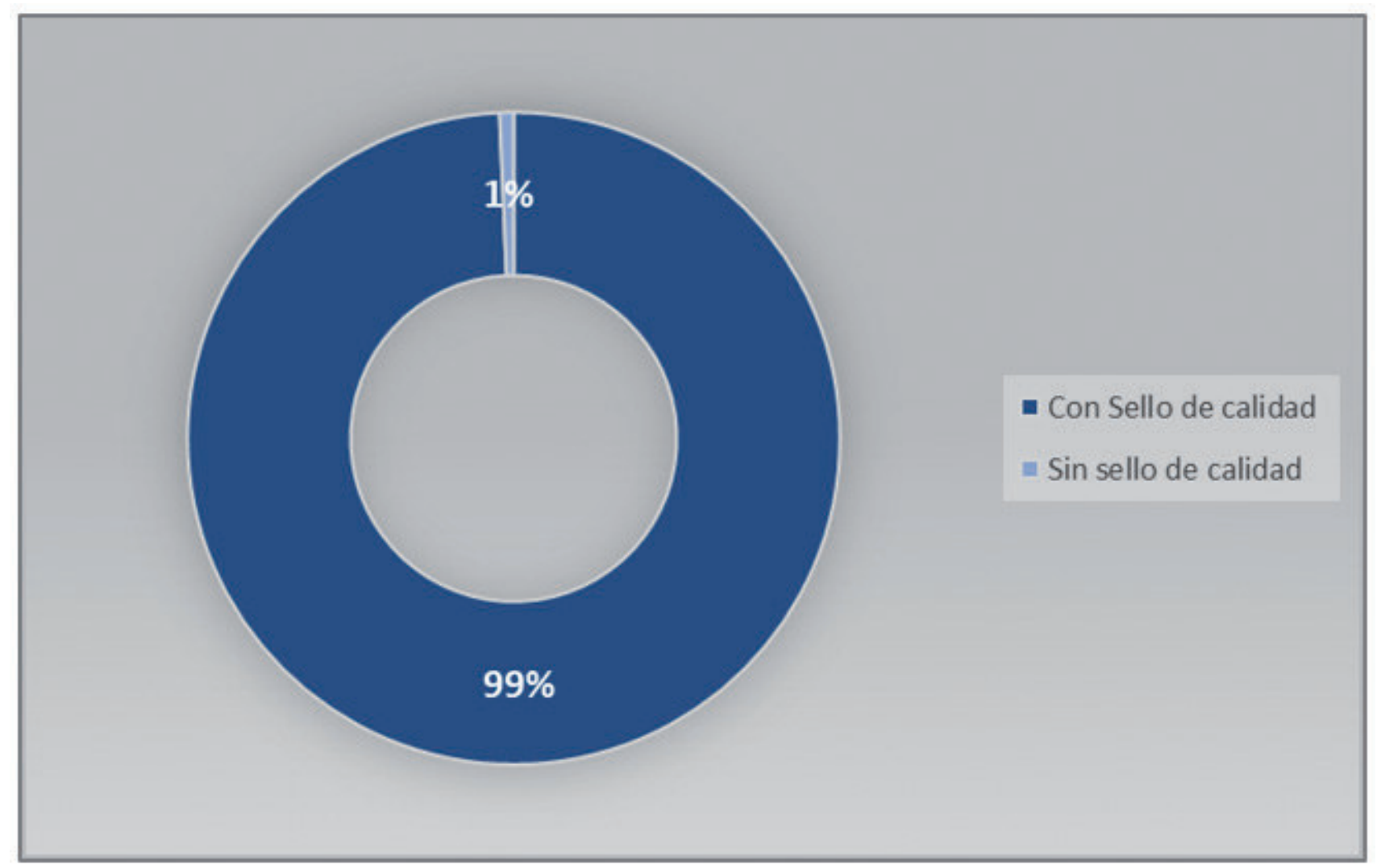

Figura 1. Distribución porcentual de las Organizaciones con sello de calidad.

Tras el primer filtrado, se procedió al estudio pormenorizado de cada una de las organizaciones a través de la observación sistemática ad hoc, se visitó todas las páginas webs de las organizaciones (Fase II) y aquellas que no presentaban páginas en internet se procedió a realización de llamada telefónica (Fase III). Después de esta fase se seleccionó una $n=35$ organizaciones de Gestión de instalaciones deportivas, que fueron las que contaban con algún tipo de certificación de calidad (antigüedad media de 25,37 años; $D T=16,1$ ), inscritas bajo personalidad jurídicas de Sociedad Limitadas $(57,1 \% ; n=20)$ y Sociedad Anónima $(42,9 \% ; n=15)$ y en su mayoría fueron privadas $(97,1 \%)$, frente a las públicas $(2,9 \%)$.El trabajo de campo se realizó por cuatro investigadores, a los cuales se le formó para la recolección de datos. Por último, se organizó y valoró los resultados para analizarlos (Fase IV). La duración del trabajo de campo se realizó durante el periodo de julio a septiembre de 2017.

\section{Resultados}

Como se puede observar a continuación (Figura 2), muestra la relación existente entre entidades con alguna certificación de calidad por comunidades autónomas de la muestra seleccionada. Solamente se observa empresas certificadas en nueve comunidades autónomas, y en la siguiente proporción: Andalucía $(n=10 ; 28,5 \%)$, Cataluña $(n=9 ; 25,7 \%)$, Madrid y País Vasco $(n=4 ; 11,4 \%)$, Islas Baleares $(n=3 ; 8,5 \%)$, Comunidad Valenciana $(n=2 ; 5,7 \%)$ y Galicia, Aragón e Islas Canarias $(n=1 ; 2,8 \%)$. 


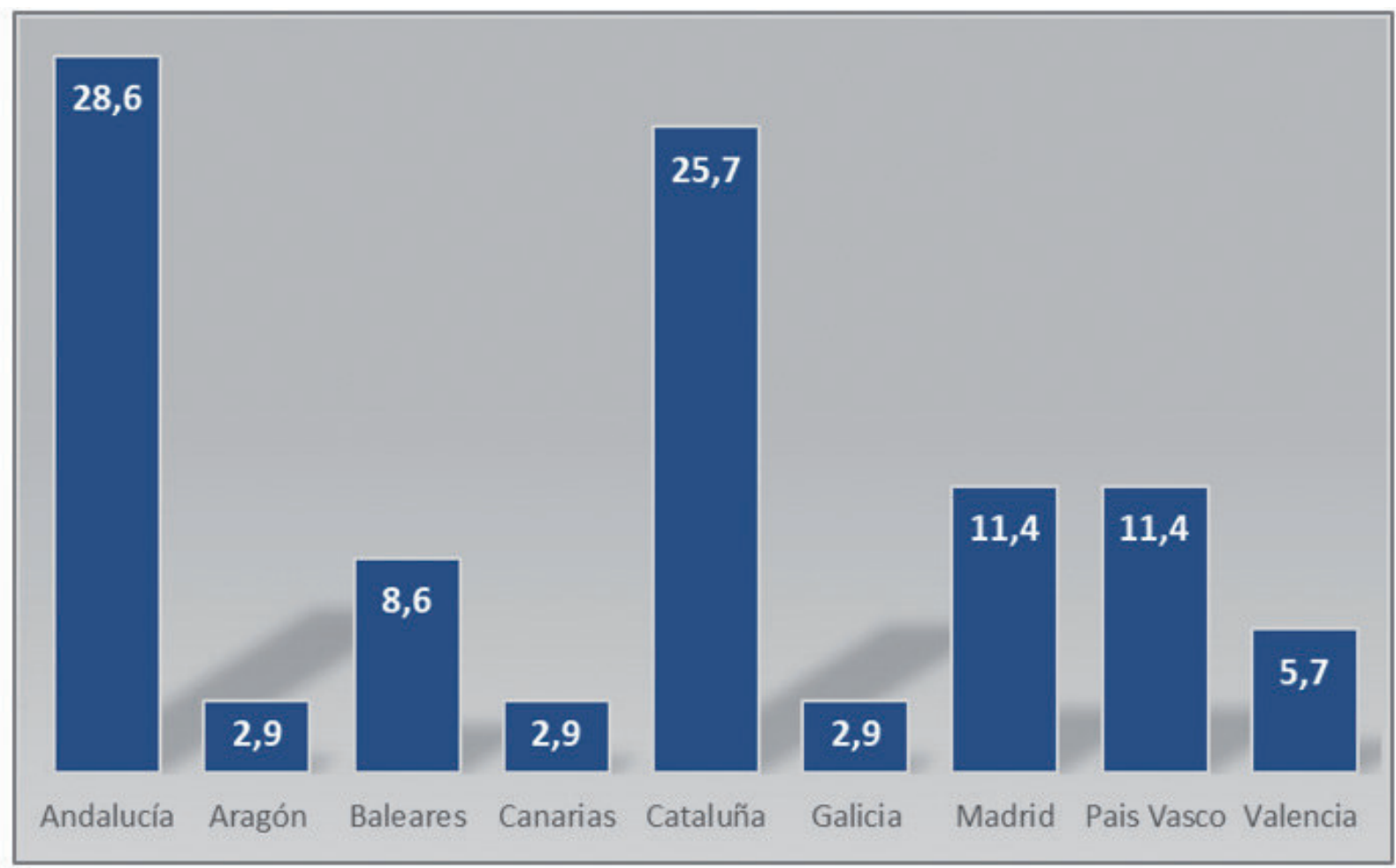

Figura 2. Relación porcentual entre certificación de calidad por comunidad autónoma.

Como se observa a continuación en el Figura 3, la relación porcentual por organizaciones y comunidades autónomas por sellos de calidad. En primer lugar, el total de certifica- ciones de calidad recogidas en las organizaciones analizadas son 62 , siguiendo la siguiente distribución ISO9001 (40,3\%), ISO14001 (35,4\%), OHSAS 18001 (17,7\%) y EFQM (6,5\%).

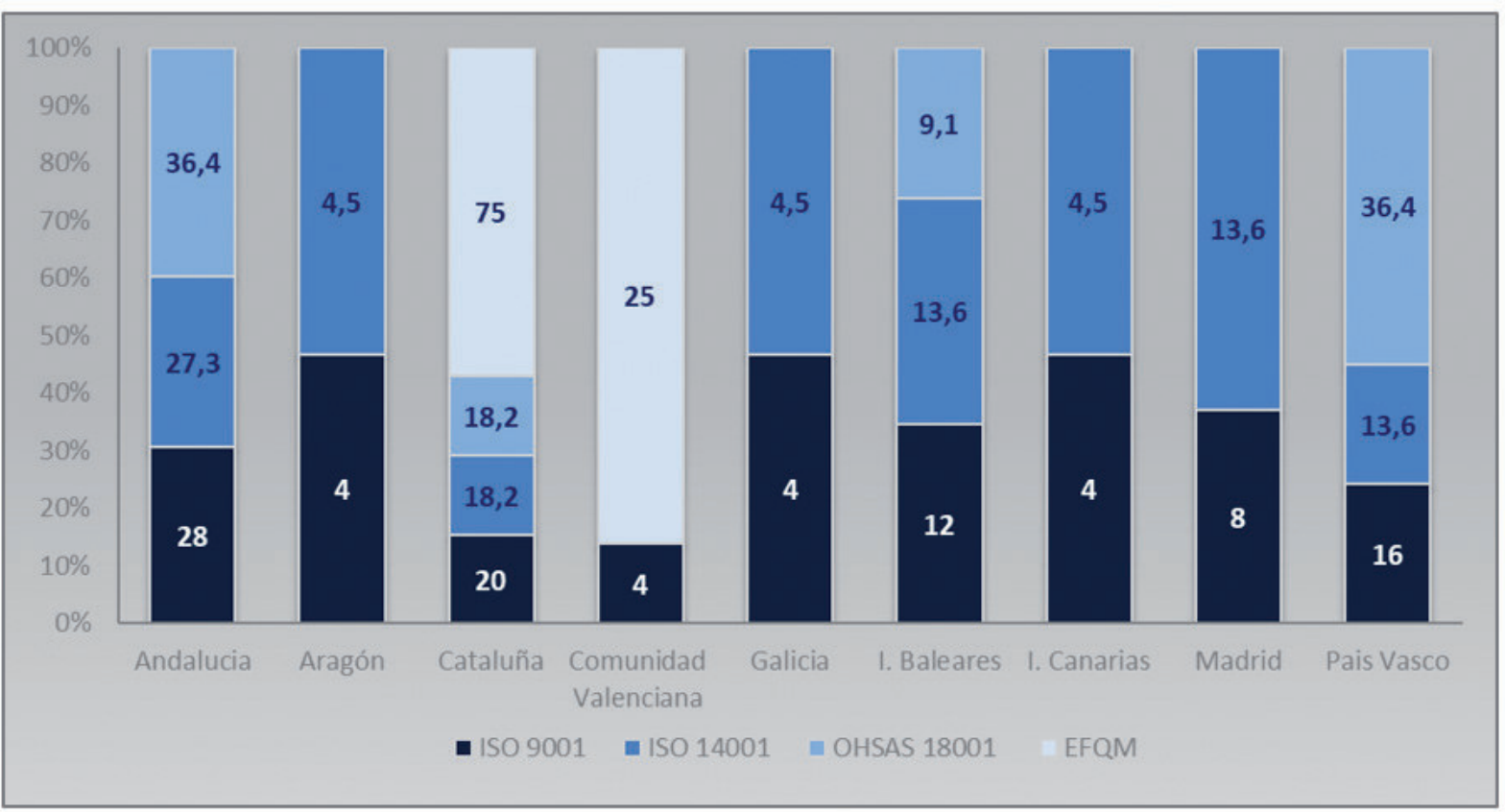

Figura 3. Relación porcentual por organizaciones y CCAA por sellos de calidad. 
Siguiendo con la relación por comunidades autónomas, se observa a la comunidad catalana, la cual es la única que cuenta con cuatro sellos de calidad entre sus organizaciones (ISO9001 $n=25 ; 20 \%$; ISO14001 $n=22 ; 18,2 \%$; OHSAS18001 $n=11 ; 18,2 \%$ y EFQM $n=4 ; 75 \%)$. Seguidamente se observa, a las comunidades Andaluza (ISO9001=36,4\%; ISO $14001=27,3 ; \quad$ OHSAS18001 $=28 \%$ ); Islas Baleares (ISO9001=12\%; ISO14001=13,6\%; OHSAS18001=9,1\%) y País Vasco con tres sellos de calidad entre sus organizaciones. Por último, y con dos sellos de calidad se encuentran las comunidades de Aragón (ISO9001=4,5\%; ISO14001=4\%); Comunidad de Valenciana (ISO9001=4\%; EFQM=25\%); Islas Canarias (ISO9001=4\%; ISO14001=4,5\%) y Madrid (ISO9001=8\%; ISO14001=13,6\%).

\section{Discusión y conclusiones}

Analizada la literatura especializada, cabe destacar la ausencia de trabajos relacionados con la certificación de calidad de las organizaciones registradas en el código de nacional de actividades económicas 9311 (Gestión de instalaciones deportivas). Los resultados obtenidos en el presente trabajo, muestran una escasa implantación de los modelos de calidad en las organizaciones de gestión de instalaciones deportivas lo cual coincide con Segarra-Vicéns (2017).

A si mismo, del análisis se desprende que el modelo de gestión más utilizado orientado hacia la calidad es el ISO9001 y gestión medioambiental ISO14001, implantado mayorita- riamente en el sector privado. Las conclusiones del presente trabajo coinciden con García-Vílchez (2008), que señala que el número de empresas certificadas al nivel mundial en la ISO 9001 es ocho veces superior al de certificadas ISO 14001 cercano a 8000.000 . Además cabe destacar la escasa implantación del modelo EFQM en el sector objeto de estudio, tendencia contraria a lo mostrado en el informe ISO Survey (2013), que indica en un estudio en 200 países certificaci lcanzar el n 4\%, lo que supone mostrado en el cerfificaciones a persar onados con la certificación de sistemas de gestión aumenta en un 4\%, lo que supone alcanzar el 1.541 .983 certificados emitidos en todo el mundo.

Como futuras líneas de investigación, se propone el análisis y situación de las organizaciones que operan según código CNAE 931 (Actividades deportivas), 9312 (Actividades de los clubes deportivos), 9313 (Actividades de los gimnasios) y 9319 (Otras actividades deportivas), por lo que se propone una nueva línea de investigación al respecto. Del mismo modo, como aplicación practicas se propone analizar el efecto sobre el rendimiento económico, ingresos por explotación y total de activos entre organizaciones con sello de calidad y aquellas que no lo tienen. Además, este trabajo contribuye al análisis del contexto del sector en organizaciones deportivas sobre la certificación de calidad. Estos resultados sirven como inicio y fundamentación teoría para futuros trabajos relacionados con la gestión de la calidad en organizaciones deportivas.

\section{Referencias}

1. Águila, C., Sicilia, A., Muyor, J., y Orta, A. (2009). Cultura posmoderna y perfiles de práctica en los centros deportivos municipales. Revista Internacional de Medicina y Ciencias de La Actividad Física y El Deporte, 9(33), 81-95.

2. Afthinos, Y., Theodorakis, N., y Nassis, P. (2005). Customers' expectations of service in Greek fitness centres. Gender, age, type of sport center and motivation differences. Managing Service Quality, 15(3), 245-258.

3. Arana-Landín, G., Heras-Saizarbitoria, I., Ochoa-Laburu C. I., y Andonegi-Martínez, J. M. (2004). Incidencia de la gestión de la calidad en los resultados de las empresas: un estudio para el caso de las empresas vascas. Revista de Dirección y Administración de Empresas, 11, 131-159.

4. Barros, C. y Gonçalves, L. (2009). Investigating individual satisfaction in health and fitness training centres. International Journal of Sport $M a-$ nagement and Marketing, 5(4), 384-395.

5. Blázquez-Manzano, A. y Feu-Molina, S. (2011). Análisis de los indicadores de calidad en materia deportiva de las cartas de servicio de las mancomunidades de municipios. Apunts. Educación física y deportes, (104), 114-126.

6. Blázquez-Manzano, A., y Feu-Molina, S. (2010). Sistema de codificación para el análisis de los indicadores de calidad de las cartas de servicios en materia deportiva. RICYDE. Revista Internacional de Ciencias del Deporte, 6(19).

7. Calabuig, F., Burillo, P., Crespo, J., Mundina, J., y Gallardo, L. (2010). Satisfacción, calidad y valor percibido en espectadores de atletismo.

Revista Internacional de Medicina y Ciencias de la Actividad Física y el Deporte, 10(40), 577-593.

8. Calabuig, F., Quintanilla, I., y Mundina, J. (2008). La calidad percibida de los servicios deportivos: diferencias según instalación, género, edad y tipo de usuario en servicios náuticos. International Journal of Sport Science, 10(4), 25-43.

9. Casadesús, F. M. y Heras Saizarbitoria, I. (2005). El boom de la calidad en las empresas españolas. Universia Business Review, 7, 90-101.

10. Cronin, J. J. y Taylor, S. A. (1992). Measuring service quality: A reexamination and extension. Journal of Marketing, 56, 55-68.

11. Cruz-Gómez, J., Lucena-Cobos, B., Méndez-Rodríguez, M., y CáceresClavero, F. (2004). Sistemas de certificación de la calidad en el sector agroalimentario Español. Distribución y Consumo, 23-39

12. Dorado-Suarez., S. (2005). Estrategias de gestión para avanzar hacia la excelencia. Madrid: Circulo de Gestores Deportivos de la Comunidad de Madrid.

13. Dorado, A. y Gallardo, L. (2005). La gestión de la calidad: el compromiso de las organizaciones deportivas para el siglo XXI. Barcelona: INDE.

14. Escanciano, G.C., Miranda, S. Esteban, J., Fernández-Sánchez, S., y Vázquez-Ordás, O. (2003). Influencia de la certificación ISO 9000 en el avance de la empresa espańola hacia la calidad total. Cuadernos de Economía y Dirección de la Empresa, 1, 99-114.

15. Espejel, J., Fandos, C., y Flavián, C. (2007). Factor clave en la satisfacción y lealtad del consumidor de aceite de oliva con DOP. Decisiones 
basadas en el conocimiento y en el papel social de la empresa: $X X$ Congreso anual de AEDEM, 1, (Ponencias), 189-201.

16. Gálvez-Ruiz, P, Grimaldi-Puyana, M., Fernández-Gavira, J. y SánchezOliver, J.A. (Enprensa). Aplicación de la teoría de la generalizabilidad en la evaluación de servicios en gestión deportiva. Sport TK Revista Euroamericana de Ciencias del Deporte.

17. Gálvez-Ruiz, P. (2011). Cuestionario para evaluar la calidad de servicios deportivos: estudio inicial de las propiedades psicométricas. Málaga: Servicio de Publicaciones de la Universidad de Málaga.

18. García-Vílchez, E.J. (2008). Ventajas de implantación de un sistema gestión ambiental. Técnica industrial, 41-43.

19. Hernández Mendo, A. (2001). Un cuestionario para evaluar la calidad en programas de actividad física. Revista de Psicología del Deporte, 10(2), 179-196.

20. ISO Survey (2013). Las certificaciones crecen un $4 \%$ en el mundo. $A E$ NOR. Revista de la normalización y la certificación, 32-35.

21. Mañas, M. A., Jiménez, G., Mayor, J. A., Martínez, V., y Moliner, C. P. (2008). Los tangibles como predictores de la satisfacción del usuario en servicios deportivos. Psicothema, 20(2), 243-248.

22. Molina-García, N. (2016). Evaluación de la calidad de servicio de centros deportivos privados. Influencia de la gestión en el bienestar de los usuarios. Universidad de Valencia: Tesis doctoral.

23. Morales Sánchez, V. (2009). Evaluación de la calidad en organizaciones deportivas: análisis de generalizabilidad. Revista de Psicología General y Aplicada, 62(1-2), 99-109.

24. Morales Sánchez, V., Blanco Villaseñor, A., y Hernández Mendo, A. (2004). Optimización de modelos de medida en la evaluación de programas de actividad física. Metodología de las Ciencias del Comportamiento, 427-433.

25. Morales-Cevidanes, M. A. (2009). La organización y gestión de las actividades físico deportivas en la Universidad: un caso práctico. Universidad de Málaga: Tesis doctoral.

26. Nuviala, A., Tamayo, J. A., Iranzo, J., y Falcón, D. (2008). Creación, diseño, validación y puesta en práctica de un instrumento de medición de la satisfacción de usuarios de organizaciones que prestan servicios deportivos. Retos. Nuevas tendencias en Educación Física, Deporte y Recreación, 14, 10-16.

27. Nuviala, A., Tamayo, J. A., Nuviala, R., González, J. A., y Fernández, A. (2010). Propiedades psicométricas de la escala de valoración de organizaciones deportivas EPOD. Retos. Nuevas tendencias en Educación Física, Deporte y Recreación, 18, 82-87.

28. Parasuraman, A., Zeithaml, V., y Berry, L. (1985). A conceptual model of service quality and its implications for future research. Journal of Marketing, 49, 41-50.

29. Parasuraman, A., Zeithaml, V., y Berry, L. (1988). SERVQUAL: A multiple-item scale for measuring consumer perceptions of service quality. Journal of Retailing, 64(1), 12-40.

30. Quintanilla, I. (2002). Psicología social del consumidor. Madrid: Pearson Educación.

31. Sánchez-Bujan, P. (2004). Hacia una Gestión de calidad en centros deportivos, la herramienta imprescindible en la gestión. Madrid: Gymnos.

32. Santos-Vijandea, M.J. y Álvarez-González, L.I. (2007). Gestión de la calidad total de acuerdos con el modelo EFQM: Evidencias sobre sus efectivos en el rendimiento empresarial. Universia Business Review, 1 , 76-89.

33. Segarra-Vicéns, E. (junio 2017). Reflexiones desde la perspectiva pública. Elementos pata el debate. Documento presentado en el 6to Congreso Iberoamericano de economía y el deporte. Ciudad Real, España.

34. Serrano, V., Rial, A., García, Ó., y Hernández Mendo, A. (2010). La evaluación de la calidad percibida del servicio como elemento clave para la gestión de los clubs de golf en España. Apunts: Educación Física y Deportes, 102(4), 95-105.

35. Theodorakis, N. D., Howat, G., Ko, Y. J., y Avourdiadou, S. (2014). A comparison of service evaluation models in the context of sport and fitness centres in Greece. Managing Leisure, 19(1), 18-35.

36. Vila, I., Sánchez, C., y Manassero, M. A. (2009). Satisfacción percibida de los usuarios de las instalaciones deportivas municipales de Palma de Mallorca. Revista Iberoamericana de Psicología del Ejercicio y el Deporte, 4(1), 59-73. 